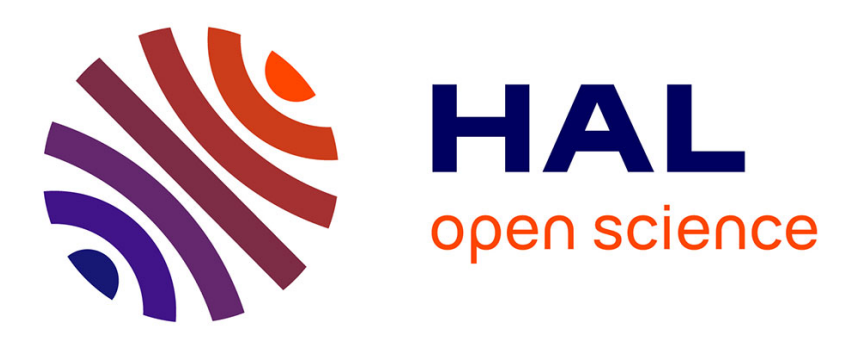

\title{
L'évaluation économique en santé au prisme de l'économie normative : principes allocatifs et règles de priorisation
}

Clémence Thebaut, Jérôme Wittwer

\section{- To cite this version:}

Clémence Thebaut, Jérôme Wittwer. L'évaluation économique en santé au prisme de l'économie normative : principes allocatifs et règles de priorisation. Revue française des affaires sociales, 2017, 1 (3), pp.169-191. 10.3917/rfas.173.0169 . halshs-02995720

\section{HAL Id: halshs-02995720 \\ https://shs.hal.science/halshs-02995720}

Submitted on 23 Mar 2021

HAL is a multi-disciplinary open access archive for the deposit and dissemination of scientific research documents, whether they are published or not. The documents may come from teaching and research institutions in France or abroad, or from public or private research centers.
L'archive ouverte pluridisciplinaire HAL, est destinée au dépôt et à la diffusion de documents scientifiques de niveau recherche, publiés ou non, émanant des établissements d'enseignement et de recherche français ou étrangers, des laboratoires publics ou privés. 


\section{L'évaluation économique en santé au prisme de l'économie normative : principes allocatifs et règles de priorisation}

Clémence Thébaut (Université de Limoges, UMR 1094, Chercheure associée LEDa-legos, Paris-Dauphine, PSL), Jérôme Wittwer (Université de Bordeaux, Bordeaux Population Health, Inserm U1219)

Revue française des affaires sociales, p. 169-191. DOI : 10.3917/rfas.173.0169. URL : https://www.cairn.info/revue-francaise-des-affaires-sociales-2017-3-page-169.htm

\section{Résumé}

Depuis 2012, en France, les industries pharmaceutiques qui sollicitent l'admission au remboursement pour un traitement innovant, sont contraintes de soumettre une évaluation économique auprès de la Commission d'évaluation économique et de santé publique (CEESP) de la Haute Autorité de Santé (HAS) afin qu'un avis sur son efficience puisse être rendu. Comme dans la plupart des pays européens, le résultat de ces évaluations est mis en perspective, dans le cadre des délibérations, avec d'autres critères de décisions plus ou moins explicites, telles que la sévérité de la maladie, l'âge des patients, les situations de fin de vie, etc. L'objectif de cet article de rapporter ces critères mobilisés par les agences pour moduler l'utilisation des ratios coût/résultat à quelques grands principes normatifs de l'économie du bien-être. Cette analyse permet d'expliciter les motivations distributives du régulateur et d'évoquer les voies possibles d'amendement du calcul économique en santé pour prendre en considération les critères de priorisation mobilisés par les agences dans leurs processus de décision, tels que la priorité accordée aux patients les plus sévèrement atteints ou encore ceux dont l'espérance de vie est la plus diminuée. L'article rend ainsi compte des possibilités pratiques proposées dans la littérature. Dans quelle mesure ces approches pourraient-elles favoriser l'acceptabilité sociale des méthodes d'évaluation économique auprès des différentes parties prenantes (décideurs, communautés médicales, patients, grand public) et accroître l'utilisation des conclusions de ces évaluations dans les décisions en matière de fixation des prix et de remboursement des produits de santé ?

\section{Introduction}

\subsection{Contexte}

Les besoins de santé sont potentiellement illimités. La diminution de la mortalité infectieuse, puis cardiovasculaire a favorisé le vieillissement de la population, qui en retour, accroît la prévalence de certaines maladies chroniques telles que les cancers (Binder-Foucard et al., 2013). Le progrès technique apporte chaque jour de nouvelles réponses à ces besoins, pour un prix élevé, ce qui participe à l'augmentation des dépenses de santé (Dormont, 2009). Le prix des traitements innovants en cancérologie comme Kadcyla (trastuzumab emtansine, Roche) ou les immunothérapies anti-PD1 (Opdivo et Keytruda) peut s'élever jusqu'à 6000€ par mois, soit un surcoût d'approximativement $100000 €$ par an pour un gain de de survie de 6 mois environ (INCA, 2016 ; Pajares y Sanchez et Saout, 2017 ; avis d'efficience de la HAS et avis rendus par le NICE sur ces spéciallités). De nombreux acteurs s'interrogent alors sur le 
montant maximum que la collectivité doit accepter de payer pour ces innovations ${ }^{1}$. En effet, dans la mesure où le financement de la santé est socialisé ( $76 \%$ des dépenses sont couvertes par l'AM obligatoire en France, cf. Drees, 2015), elles rentrent en concurrence avec d'autres investissements publics qui font également l'objet d'attentes sociales fortes: l'éducation, l'environnement, la prise en charge des personnes dépendantes, la lutte contre la pauvreté et l'exclusion, etc. La croissance des dépenses de santé doit donc être maîtrisée de sorte que le niveau de ces dépenses soit cohérent avec la valeur que la collectivité accorde à la santé, par rapport aux autres enjeux sociaux. Nous sommes donc contraints de choisir, parmi l'ensemble des stratégies qui sont disponibles (médicaments, dispositifs, programmes de santé publique, interventions non médicamenteuses), celles qui seront financées collectivement.

Pendant longtemps, en France, le processus de fixation des prix était, officiellement, principalement fondé sur l'évaluation de l'efficacité clinique des traitements (appréciée à travers la notion de Service médical rendu et d'Amélioration du service médical rendu) par la Commission de la transparence de la Haute Autorité de Santé (HAS) ${ }^{2}$. Il est apparu progressivement que ce critère n'était pas suffisant pour garantir une gestion efficiente du panier de soins remboursables (Cour des comptes, 2011). La loi de financement de l'assurance maladie de 2012 a alors imposé aux industriels d'apporter à la HAS une information sur le ratio différentiel coût/résultat (RDCR) associé au produit, lorsqu'ils sollicitent le remboursement d'un traitement innovant (Midy et al., 2015). Le résultat est la plupart du temps exprimé en coût/année de vie en bonne santé c'est-à-dire en coût/QALY dans le jargon de l'évaluation médico-économique. La robustesse du modèle médico-économique est évaluée par la Commission d'évaluation économique et de santé publique (CEESP) de la HAS et l'avis qu'elle émet sur le résultat de l'évaluation est transmis au Comité économique des produits de santé (CEPS) afin de servir aux négociations du prix. Si une stratégie est à la fois moins chère et plus efficace, il n'y pas de difficulté à interpréter le résultat de l'évaluation, elle est efficiente. En revanche si elle est à la fois plus coûteuse et plus efficace, il faut s'assurer, pour pouvoir conclure sur son efficience, que la collectivité accorde au moins autant de valeur aux bénéfices de santé qu'apporte la technologie, qu'aux résultats obtenus en utilisant autrement les ressources consommées dans le secteur de la santé ou dans d'autres secteurs (coût d'opportunité). Pour porter un tel jugement, les économistes de la santé et les acteurs institutionnels proposent souvent de recourir à une " valeur de référence » (KCE, 2009). Cette valeur représente le montant maximum que la collectivité est prête à dépenser pour gagner une année de vie en bonne santé supplémentaire. En France, la HAS a explicitement souligné l'intérêt qu'il y avait à utiliser une valeur de référence tout en rappelant les difficultés que pose sa définition. Elle appelait l'organisation d'un débat démocratique sur le sujet (HAS, 2014).

L'une des principales questions que soulève la définition d'une valeur seuil est de savoir si les gains de santé ont toujours la même valeur ou si ceux-ci sont susceptibles de varier en fonction des circonstances personnelles des patients ou des caractéristiques de la maladie, par exemple en fonction du degré de sévérité de la maladie, de l'âge des patients et de leur espérance de vie, de leur vulnérabilité, etc. Comme l'ont montré Sabik et Lie (2008), Raftery,

\footnotetext{
1 UFC que choisir (https://www.quechoisir.org/actualite-nouveaux-anticancereux-les-prix-donnent-la-nauseen1691/), appels de 101 cancérologogues (http://sante.lefigaro.fr/actualite/2016/03/14/24737-lappel-110cancerologues-contre-cout-traitements).

2 Dans les pratiques, les laboratoires pouvaient, avant la LFSS de 2012, présenter au CEPS des valeurs de ratio différentiel coût-résultat dans le cadre des négociations confidentielles menées sur la fixation du prix de leur produit. Nous remercions un rapporteur de cet article d'avoir apporté cette précision.
} 
(2009), Golan et al. (2011) et la HAS (2014), les agences combinent toutes les critères d'efficacité et d'efficience avec d'autres critères, le plus souvent liés aux caractéristiques de la maladie (sévérité, fardeau de la maladie, nécessité du traitement, etc.). Elles ont recours à des procédures variées.

- Certaines agences refusent de définir une valeur de référence et apprécient le niveau du RDCR associé à chaque stratégie, au cas par cas (comme c'est le cas actuellement en France, Suède, Danemark, Norvège, Belgique, Australie et Nouvelle-Zélande), en explicitant ou non des critères de priorisation.

- D’autres agences utilisent une valeur de référence et prévoient a priori les contextes dans lesquels il est permis d'y déroger, comme par exemple le National Institute for Health and Care Excellence (NICE) en Grande-Bretagne depuis 2009 pour les traitements de fin de vie (Collins, 2013). Les comité d'évaluation des technologies de santé (Technology Appraisal Comittee) du NICE sont en effet libres de recommander le remboursement des traitements associés à un RDCR supérieur à la valeur seuil communément admise ( $£ 20000-£ 30000 / Q A L Y$ ) lorsque les traitements augmentent la survie d'au minimum trois mois, pour des patients dont l'espérance de vie est inférieure à 24 mois, lorsque la taille de la population cible est réduite (NICE, 2009).

- Elles peuvent enfin utiliser différentes valeurs de référence en fonction des circonstances. Le NICE a par exemple un temps envisagé de remplacer la procédure de dérogation pour les traitements de fin de vie, qui ne prévoit pas de valeur seuil maximum, par la définition de différentes valeurs seuil de référence en fonction de l'impact de la maladie sur l'espérance de vie. La valeur seuil varie alors entre $£ 20000$ et $f 50000 / Q A L Y e$ (NICE, 2014). De même la valeur seuil est établie à $f 100000$ dans le cadre des maladies ultra orphelines ${ }^{3}$.

Ces procédures ont toutes en commun de remettre en question la célèbre formule de Weinstein " $A Q A L Y$ is a $Q A L Y$ is a $Q A L Y$ » (1988), c'est-à-dire qu'elles remettent en question le principe utilitariste benthamien selon lequel " chacun compte pour un et personne pour plus $d^{\prime} u n$ ». Pour autant, aucune institution n'amende le calcul économique en ce sens.

\subsection{Objectif}

L'objet de cet article est de rapporter les critères de priorisation mobilisés par les agences pour moduler l'utilisation des ratios coût/résultat à quelques grands principes normatifs de l'économie du bien-être. Nous y voyons un double intérêt. Le premier est d'expliciter et de formaliser les motivations distributives du régulateur et éventuellement d'en souligner les contradictions ou les points de tension. Le second est d'évoquer les possibilités pratiques de mise en œuvre d'un calcul économique en santé intégrant les priorités que le système de santé souhaite concrétiser. II est en effet d'usage courant en économie de considérer des dimensions distributives dans la formulation des objectifs de bien-être social.

Les travaux sur les questions de la pondération des QALYs sont nombreux et anciens. II ne s'agit pas dans cet article de développer de nouvelles propositions dans ce sens, mais de proposer une discussion formelle des voies possibles d'amendement du calcul économique en santé permettant de prendre en considération les critères de priorisation mobilisés par les agences dans leurs processus de décision.

\footnotetext{
${ }^{3}$ https://www.nice.org.uk/about/what-we-do/our-programmes/nice-guidance/nice-technology-appraisalguidance/consultation-on-changes-to-technology-appraisals-and-highly-specialised-technologies
} 


\subsection{Plan}

Les discussions sur les principes de répartition des ressources collectives se concentrent autour de trois principales questions (Sen, 1987 ; Van Parjis, 1991 ; Fleurbaey 1996). Cette typologie est utilisée pour organiser les principes redistributifs utilisés implicitement par les agences lorsqu'elles mobilisent les critères de sévérité et de fardeau de la maladie pour prioriser l'allocation des ressources en santé, sachant que ceux-ci seront spécifiquement analysés dans le cadre de cet article. La sévérité de la maladie est mesurée en fonction l'impact de la maladie sur la qualité de vie et/ou l'espérance de vie des patients. Le fardeau de la maladie est quant à lui mesuré en estimant la différence d'espérance de vie en bonne santé pour les patients de même âge, avec ou sans la maladie.

- La $1^{\text {er }}$ question concerne le principe de répartition du distribuendum (objet que l'on souhaite répartir équitablement dans la population): cherche-t-on à répartir les ressources de façon à maximiser la somme des quantités produites de ce distribuendum dans la population ou cherche-t-on à les répartir de façon à réduire la dispersion interindividuelle dans la population ? On constate ainsi que les critères de sévérité et de fardeau de la maladie mobilisent un même principe de répartition fondé sur un objectif d'égalisation du distribuendum qui contraste avec l'utilisation d'une valeur de référence unique, sans pondération des résultats de santé (section 2).

- la $2^{\mathrm{e}}$ question concerne la définition ce que l'on souhaite répartir équitablement (distribuendum) : cherche-t-on à répartir équitablement des conditions objectives de vie, tels que le revenu, l'éducation, la santé, c'est-à-dire extérieures à l'individu les appréciant, ou cherche-t-on à répartir équitablement un niveau d'appréciation subjective des situations individuelles (utilité, satisfaction, bien-être) ${ }^{4}$ ? Par ailleurs, ce distribuendum est-il complet ou porte-t-il sur des dimensions particulières des situations ? Comme nous le verrons, les critères de priorité utilisés par les agences impliquent une évaluation des situations individuelles exclusivement sanitaires (section 3). Elles n'envisagent pas d'évaluer l'impact d'interventions de santé sur les conditions de vie globales des personnes comme le proposent aujourd'hui les théoriciens de la justice sociale tels que Sen et Fleurbaey.

- la $3^{\mathrm{e}}$ question concerne l'évaluation du distribuendum : cherche-t-on à répartir équitablement les chances d'accéder au résultat ou l'atteinte effective du résultat. Cette distinction est importante pour comprendre la différence entre l'approche par les capabilités développée par Sen et celle par le revenu équivalent santé développée par Fleurbaey qui ont chacune fait l'objet d'applications concrètes dans le cadre de l'évaluation économique des technologies de santé. La première vise à égaliser les opportunités de fonctionnements, tandis que la seconde vise à égaliser les résultats sur des dimensions de la vie des individus jugées socialement fondamentales (comme la santé par exemple). Elles constituent toutes les deux des pistes privilégiées pour amender le calcul économique en santé de sorte qu'il intègre des principes redistribustifs (section 3.3).

\section{Le principe de répartition du distribuendum sous-jacents aux différents critères de priorisation des agences : critères de sévérité et fardeau de la maladie}

\footnotetext{
${ }^{4}$ Sur les enjeux entre l'évaluation objective et subjective des situations individuelles, nous renvoyons à Clément (2009), Fleurbaey (2003) et Hurka (1996, p. 1437).
} 
Le principe de répartition retenu lorsque les agences utilisent un critère d'efficience dans le cadre de processus de fixation de prix et de remboursement des produits de santé est conforme à celui retenu dans le modèle de justice utilitariste. Le principe utilitariste du calcul économique en santé s'exprime par le fait que l'efficacité d'un traitement est évaluée par la somme des gains individuels. Il apparaît encore plus immédiatement si l'on raisonne à budget donné, c'est-à-dire en considérant comme donné le budget couvrant le financement de l'ensemble des traitements. Dans ce cas, le calcul économique en santé équivaut à maximiser sous contrainte la somme des utilités liées à la santé, soit à maximiser la fonction " utilitariste » $: W_{U}=\sum_{i} q_{i}$.

En effet, le calcul économique en santé évalue un nouveau traitement en confrontant la valeur de la somme des gains en santé individuels qu'il permet à son coût, par rapport aux stratégies disponibles. Plus formellement, un traitement est jugé efficient si : $\lambda \sum_{i} \Delta q_{i} \geq \Delta C$. $^{5}$

$q_{i}$ représente l'utilité liée à la santé (exprimé en QALY) et $\Delta q_{i}$ le gain d'utilité permis par le traitement considéré pour l'individu $i ; \Delta C$ désigne le surcoût entrainé par l'adoption du nouveau traitement et $\lambda$ est la valeur monétaire de référence, le prix que la société est prête à payer pour un gain d'une année de vie en bonne santé. $q_{i}$ est dans la pratique du calcul économique en santé définie par la somme ${ }^{6}$ des années de vie pondérées par la qualité : $q_{i}=$ $\sum_{t=0}^{T} q_{i}^{t}$ où $q_{i}^{t}$ désigne la qualité de vie à l'année $t$. Elle est normée par la bonne santé et la mort : $q_{i}^{t}=1$ représente une année de vie en bonne santé (un QALY) et $q_{i}^{t}=0$ la mort ${ }^{7}$.

Tous les gains individuels ont le même poids et le gain unitaire est égal au gain moyen. Chacun a le droit de bénéficier d'un traitement, à condition qu'il ait été démontré que les ressources consommées ne peuvent pas être utilisées à meilleur escient ailleurs en produisant davantage de gains en santé. Les ressources en santé sont donc réparties en fonction de la "capacité à bénéficier des individus " (Culyer et Wagstaff, 1992).

Considérer, explicitement ou implicitement, des valeurs de références différentes en fonction des situations comme le font de nombreuses agences revient à considérer que les gains en QALY n'ont pas la même valeur selon les patients. C'est une négation du principe utilitariste du calcul économique en santé fondé sur la sommation des gains en santé. Traduire cette pratique dans un cadre à budget donné supposerait de ne plus maximiser une fonction définie comme la somme des utilités (liées à la santé) mais comme une somme pondérée des utilités.

\subsection{Les pratiques des agences en matière de critères de priorisation}

Le critère de répartition de type utilitariste est en effet contourné par les agences qui modifient la valeur de référence pour certains types de patients, en particulier ceux qui sont dans les états de santé les plus sévères et/ou ceux dont l'espérance de vie est le plus réduite. Comme mentionné ci-dessus, le NICE admet des dérogations à la valeur seuil pour les traitements de fin de vie (NICE 2008, 2009). Certains auteurs comme Raftery avaient depuis longtemps montré l'hétérogénéité des avis rendus par le NICE selon les caractéristiques de la

\footnotetext{
${ }^{5}$ Cette inégalité se réécrit classiquement en ratio unitaire différentiel coût/résultat (RDCR) : $\frac{\Delta C / n}{\sum_{i} \Delta q_{i} / n} \leq \lambda ; n$ désignant le nombre d'individus traités.

${ }^{6}$ Par souci de simplification nous omettons ici d'actualiser les utilités comme il est d'usage dans le calcul économique en santé pour les coûts et les utilités.

${ }^{7}$ Sans vouloir ici entrer dans le détail des méthodes d'évaluation des QALY et de leurs limites, rappelons qu'ils sont définis comme des utilités (liées aux états de santé) valorisées par les préférences individuelles. Au regard de la classification précédente, il s'agit d'une valorisation subjective moyenne d'un attribut objectif (l'état de santé). Notons que l'on peut définir des états de santé pires que la mort dont l'utilité est négative.
} 
maladie (2005). Aux Pays-Bas, l'agence nationale d'évaluation des technologies de santé, le $C V Z$, indique pouvoir recommander le remboursement d'une technologie dont le ratio coût/efficacité est supérieur à $80000 € / Q A L Y$ lorsque le décès des patients est imminent en l'absence de traitement ou leur qualité de vie très dégradée : "Patients would die immediately if given no treatment or whereby patients without treatment would have avery low quality of life " (CVZ, 2010). Enfin en Australie, le Pharmaceutical Benefits Advisory Committee (PBAC, 2013) limite la portée du critère d'efficience pour les patients dont la pathologie est sévère et progressive et lorsqu'elle les expose à un décès prématuré : "The medical condition defined by the requested restriction is severe, progressive and expected to lead to premature death" (PBAS, 2013, p.258). On constate par ailleurs que, ces dernières années, les économistes de la santé se sont davantage intéressées à ces critères et qu'ils ont mené différentes enquêtes pour expliciter l'importance que leur accordent les individus en population générale. L'équipe du SCHarr de Sheffield, par exemple, a réalisé pour le NICE des enquêtes en population générale pour identifier les groupes d'individus considérés comme prioritaires : ceux dont le fardeau de la maladie est le plus élevé, les patients en fin de vie ou ceux susceptibles de bénéficier au maximum des traitements (Brazier et al., 2013). Une enquête similaire a été réalisée par le KCE en Belgique auprès du grand public et auprès d'acteurs institutionnels (Cleenput, 2014). L'objectif était, à terme, d'utiliser le résultat de ces enquêtes pour introduire des pondérations dans le calcul économique en fonction des circonstances. A notre connaissance, ces enquêtes n'ont pas encore conduit ces agences à faire évoluer les critères de priorisation.

En définitive, il apparaît dans les exemples ci-dessus que le critère de sévérité de la maladie est quasi-systématiquement pris en compte par les agences. Celui de fardeau est également largement discuté même s'il est finalement peu utilisé en pratique. Le NICE, en GrandeBretagne, avait très sérieusement envisagé de mobiliser le critère de fardeau de la maladie pour justifier d'élever le niveau de la valeur de référence, pour finalement y renoncer. II proposait de prendre en compte à la fois le fardeau absolu de la maladie et le fardeau relatif. Comme mentionné ci-dessus, le fardeau absolu correspond à la différence d'espérance de vie en bonne santé pour les patients de même âge, avec ou sans la maladie. Le fardeau relatif est une variante de la notion de fardeau absolu utilisée aux Pays-Bas (van de Wetering, 2013). II correspond à la proportion de l'espérance de QALY que la maladie fait perdre au patient par rapport à l'espérance de QALY dont bénéficie un individu du même âge dans la population générale (Towse, Barnsley, 2013 ; Kusel, 2015). Tandis que le critère de fardeau de la maladie "absolu" accorde une forte priorité aux populations les plus jeunes, cette priorité est presque nulle avec le critère de fardeau de la maladie " relatif ".

Les critères de sévérité et fardeau de la maladie justifient d'améliorer en priorité la situation des individus les plus malades, c'est-à-dire de réduire les inégalités en matière d'états de santé. Plus l'état de santé de départ d'un individu est dégradé, plus son amélioration est valorisée par la collectivité. En fonction du degré d'aversion aux inégalités, la disposition à payer collective pour gagner une unité de santé supplémentaire varie en fonction du niveau de départ. Il est possible conceptuellement d'adapter le calcul de l'économie du bien-être à ces préoccupations redistributives (Encadré 1). Ce faisant, nous nous écartons bien sûr du cadre théorique welfariste orthodoxe, comme s'en écartent déjà les économistes de la santé avec l'utilisation des QALY (Forget, 2004).

2.2. Les possibilités pratiques proposées dans la littérature pour intégrer des principes de répartition égalitaristes 
Différents auteurs ont proposé d'intégrer des principes de répartition égalitaristes dans l'évaluation économique en utilisant des coefficients pour pondérer les bénéfices de santé en fonction de l'état de santé de départ (avant l'intervention). Différents ensembles de coefficients peuvent être proposés par les évaluateurs en fonction du degré d'aversion aux inégalités (Nord, 1999) ou en fonction du sacrifice que la collectivité est prête à consentir en termes de gains de santé totaux pour égaliser leur répartition au sein de la population (Williams, 1997). Des ensembles peuvent également être définis à partir des préférences de la population générale en matière de principes redistributifs (Wailoo et al. 2009). En règle générale, dans ces approches, seuls les bénéfices de santé sont pondérés et non les coûts, le résultat s'exprime donc sous la forme d'un ratio différentiel coût/résultats pondérés. Dans les travaux les plus récents, il est proposé d'agréger l'ensemble des conséquences de l'intervention au niveau individuel, à la fois en termes de coûts et de résultats à l'aide d'une fonction de bien-être sociale (Asaria et al. 2016; Samson et al. 2017). Ces méthodes permettent de prendre en compte de façon plus extensive les conséquences redistributives des interventions. Asaria et al. utilisent comme indice l'espérance d'année de vie ajustée sur la qualité de vie (QALE) : les coûts d'opportunité sont convertis en QALY au moyen de la valeur seuil retenue en Grande-Bretagne (f20000/QALY) (pour une revue plus détaillée de ces méthodes cf. Cookson et al., 2017).

Encadré 1: Le critère de sévérité de la maladie dans le calcul économique

Dans le cadre formel présenté plus haut, tenir compte de la sévérité de la maladie se traduit dans le calcul économique par le recours à la fonction de bien-être social suivante: $W_{S}(q)=\sum_{i} f\left(q_{i}\right)$ avec $f^{\prime}>0$ et $f^{\prime \prime}<$ 0 .

L'aversion à l'inégalité traduite par la concavité de la fonction $f$ traduit une aversion à l'inégalité de la distribution des espérances de vie en bonne santé. Si la sévérité de la maladie est définie par une espérance de vie réduite, la fonction $W_{S}$ ainsi définie rend bien compte du critère de sévérité comme critère d'allocation des ressources du système de santé en surpondérant les gains en QALYs des individus à l'espérance de vie en bonne santé la plus faible. La fonction $W_{S}$ peut être comprise comme un instrument de pondération des QALYs dans le calcul économique : pour deux individus définis par les utilités $q_{1}$ et $q_{2}\left(q_{1}<q_{2}\right)$, le poids relatif des gains en QALYs de l'individu 1 par rapport à ceux de l'individu 2 sont dans un rapport égal au rapport de utilités sociales marginales : $\frac{W^{\prime}{ }_{S}\left(q_{1}\right)}{W^{\prime}{ }_{S}\left(q_{2}\right)}(>1)$.

\section{Le choix du distribuendum sous-jacent aux différents critères de priorisation des agences}

\subsection{Le distribuendum induit par les critères de sévérité et de fardeau de la maladie}

On a montré que les agences introduisaient des critères de priorisation dans leurs usages du calcul économique, et principalement deux critères : celui de la sévérité de l'état de santé et celui du fardeau de la maladie qui impliquent chacun un critère de répartition de type égalitariste. Les deux critères n'impliquent toutefois pas un distribuendum identique. La priorité accordée aux patients les plus sévères vise à répartir équitablement des niveaux d'état de santé (capacité fonctionnelle, absence de douleur, absence de pronostic vital engagé). La priorité accordée aux patients dont le fardeau de la maladie est le plus élevé vise à répartir équitablement une durée de vie donnée corrigée de la qualité de vie. Les distribuendum sont objectifs ou partiellement objectifs lorsque la sévérité ou le fardeau de la maladie est estimé au moyen de QALY qui mesurent la qualité de vie des patients dans les différents états à partir des préférences individuelles liées à la santé (Brazier et al., 2007) (Cf. note 6). En outre, ces distribuendum ne prennent en compte qu'une dimension particulière de la vie des individus, leur santé. 
Le critère de priorité fondé sur la notion de fardeau de la maladie est le plus souvent justifié par les réflexions philosophiques de Harris, développé ensuite par Williams (Miners et al. 2013). L'idée selon laquelle il conviendrait de garantir à tous une durée de vie donnée a d'abord été proposé, d'un point de vue philosophique, par Harris avec l'argument du fair innings (1985), puis par Williams (1997). Selon Harris, tout individu qui décèderait prématurément subit une injustice que la collectivité est justifiée à prévenir. Il est pertinent d'allouer en priorité les ressources de façon à maximiser les chances que tous atteignent un âge donné. Harris ne précise cependant pas quel doit être cet âge de référence, ni la méthode pour le définir. Selon lui, il n'est pas possible de distinguer différents degrés d'injustice en fonction de l'âge du décès. Dès lors qu'ils n'ont pas encore atteint ce fair innings, ils sont tout autant pénalisés qu'ils aient 20, 30 ou 40 ans. Williams, en revanche, propose de définir l'âge de référence en s'appuyant sur l'espérance de vie moyenne à la naissance dans la population générale. Contrairement à Harris, il considère que l'injustice que subit l'individu qui décède prématurément croît en fonction de l'écart entre l'âge du décès et cette espérance de vie moyenne. Enfin il recommande de prendre en compte la qualité de vie sur tout le cycle de vie de l'individu ce qui soulève des difficultés informationnelles importantes ${ }^{8}$. L'encadré 2 présente les différences entre les critères de fair innings, de fardeau de la maladie et de sévérité en utilisant un formalisme welfariste plus précis permettant de mettre en évidence la nature différente du distribuendum et ses conséquences en termes de choix collectifs ${ }^{9}$. La priorisation en fonction du fardeau n'est pas nécessairement équivalente à la priorisation en fonction de la sévérité de la maladie. En effet, à sévérité donnée de la maladie, un patient jeune, souffrant donc d'un fardeau plus élevé, bénéficiera d'une pondération de ses gains en QALYs plus important qu'un patient plus âgé si l'on adopte une priorisation en fonction du fardeau (ce qui ne serait naturellement pas le cas dans le cas d'une priorisation en fonction de la sévérité).

Nous discutons plus bas de la possibilité de concilier ou non ces critères. Le premier avantage du formalisme welfariste est d'expliciter les présupposés éthiques du choix social. Dans le cas présent, il permet de mettre en évidence la tension entre l'objectif du fair innings et de celui de la priorisation des patients sévères à l'espérance de vie en bonne santé réduite. Cette tension est une réalité du calcul économique en santé qui n'a pas trouvé aujourd'hui d'issue claire.

Encadré 2: Les critères de fair innings et de fardeau de la maladie dans le calcul économique

Le critère de fair innings tel que le conçoit Williams impose de considérer l'état de santé sur l'ensemble du cycle de vie et non sur les années restant à vivre. Dans la formalisation que nous retenons cela supposerait de modifier la définition de l'utilité liée à la santé en définissant l'utilité sur l'ensemble du cycle de vie: $Q_{i}=$ $\sum_{t=-N}^{T} q_{i}^{t}$ où $N$ désigne le nombre d'années déjà vécues par l'individu $i$. La fonction de bien-être social s'écrit alors ainsi: $W_{F I}(Q)=\sum_{i} f\left(Q_{i}\right)$. Cette reformulation rencontre une difficulté majeure de nature informationnelle. L'évaluation de l'utilité liée à l'état de santé sur l'ensemble du cycle de vie demande de collecter des données rétrospectives difficiles à obtenir dans la pratique.

Pour cette raison, c'est la notion de fardeau de maladie qui est, en pratique, souvent mobilisée ; le fardeau est défini comme la perte d'année de vie en bonne santé affectant l'individu $i: S_{i}=\left[\bar{q}_{i}-q_{i}\right]$, où $\bar{q}_{i}$ est définie comme l'espérance de vie en bonne santé d'un individu de même âge que l'individu $i$ et ne souffrant pas de

\footnotetext{
${ }^{8}$ Sur les différentes versions du fair innings Cf. Tsuchiya, 2001

${ }^{9}$ La priorité accordée aux patients atteints de maladies rares par les différentes agences (ex. le NICE avec la commission spécialisée sur les Highly specialised technologies (cf. note 3) ou encore le programme Life Saving Drug Program en en Australie) est en partie prise en compte par le critère de fair innings dans la mesure où ces maladies sont le plus souvent des maladies congénitales, handicapantes, ayant des conséquences à la fois sur la qualité et l'espérance de vie des patients.
} 
la maladie affectant $i$. On peut alors redéfinir l'objectif social comme la minimisation de la somme des fardeaux, ce qui conduit à la maximisation de la fonction de bien-être social suivante : $W_{f m}=-\sum_{i}\left[\bar{q}_{i}-q_{i}\right]$ (ce qui est équivalent à la minimisation de la somme des fardeaux). On constate que cette fonction définit le même objectif que la fonction $W_{S}$ définie sur l'utilité liée à la santé (sévérité de la maladie, encadré 1 ) ; on peut effet réécrire la fonction $W_{f m}$ ainsi : $W_{f m}=\sum_{i} q_{i}-\sum_{i} \bar{q}_{i}$. En revanche, si l'on considère une fonction de bien-être social qui pondère les gains d'utilité en fonction du fardeau de la maladie le choix du distribuendum devient déterminant. Ainsi, la fonction $W_{F M}=-\sum_{i} F\left[\bar{q}_{i}-q_{i}\right]$, avec $F$ une fonction croissante convexe, surpondère les gains en utilité des personnes souffrant d'un fardeau élevé et non d'une espérance de vie en bonne santé réduite (même si bien entendu les deux dimensions ne sont pas indépendantes). La fonction $W_{F M}$ permet de rendre compte de l'idée du fair innings en surpondérant les gains en santé des personnes dont la maladie est la plus pénalisante en termes d'espérance de vie en bonne santé. Elle permet donc de favoriser l'égalisation de l'espérance de vie en bonne santé même si elle ne considère pas la qualité de vie de manière rétrospective comme le demanderait une analyse en fair innings à l'instar de la fonction $W_{F I}$. L'usage du fardeau de la maladie pour pondérer les gains en santé suppose de considérer explicitement l'âge des patients. Ainsi, pour une espérance de vie en bonne santé donnée, un gain en santé prendra socialement d'autant plus de valeur que le patient considéré est jeune. Le fardeau de la maladie comme distribuendum rompt ainsi avec l'égalité devant l'état de santé dans le sens où pour des patients qui font faces à la même espérance de vie en bonne santé les gains en santé n'auront pas le même poids pour l'un et pour l'autre. C'est en cela que son usage dans le calcul économique en santé est discuté. Les deux distribuendum, sévérité de la maladie et fardeau de la maladie, poursuivent des logiques éthiques distinctes qui conduisent à des allocations des ressources qui peuvent être sensiblement différentes.

\subsection{Peut-on prendre en compte en même temps sévérité et fair innings ?}

II est délicat de rendre compte simultanément dans une fonction de bien-être des objectifs d'égalisation des espérances de vie en bonne santé et de priorisation des personnes les plus sévèrement atteintes (cf. Encadré 3). Dans cet esprit le NICE proposait l'usage du fardeau relatif, $F R_{i}=\left(\bar{q}_{i}-q_{i}\right) / \bar{q}_{i}$, comme facteur de pondération des gains en santé (ou comme distribuendum pour reprendre notre vocabulaire) dans le but d'intégrer la notion de fair innings dans le calcul économique en santé sans pénaliser pour autant trop fortement les gains en santé pour les patients âgés (Towse, Barnsley, 2013 ; NICE, 2014).

Encadré 3 : Compatibilité formelle entre le critère de fair innings et de fardeau de la maladie

D'un point de vue formel on peut proposer de définir le fardeau de la maladie comme un fardeau social en considérant la priorité donnée aux patients sévères : $F S_{i}=\left[f\left(\bar{q}_{i}\right)-f\left(q_{i}\right)\right]$ avec $f$ une fonction croissante concave. La fonction objectif se réécrit alors : $W_{F S}=-\sum_{i} F\left[f\left(\bar{q}_{i}\right)-f\left(q_{i}\right)\right]_{-}$avec $F$ une fonction croissante convexe comme précédemment (sa maximisation équivaut à minimiser la somme des fardeaux sociaux). Pour un niveau donné du fardeau social, le gain en santé est d'autant plus valorisé que le patient est sévèrement malade. La fonction $f$ joue ici un rôle équivalent à celui joué dans la fonction $W_{S}$. Elle joue cependant ici un second rôle en déterminant la valeur du rapport $\rho=\left(f\left(\bar{q}_{i}\right)-f\left(q_{i}\right)\right) /\left(\bar{q}_{i}-q_{i}\right)$ comparant le fardeau social au fardeau de la maladie. On peut ainsi imaginer que pour des valeurs faibles le l'espérance de vie en bonne santé $\left(\bar{q}_{i}\right)$ le fardeau social de la maladie soit supérieur au fardeau $(\rho>1)$ pour atténuer la sous pondération (induite par la convexité de $F$ ) des gains en santé pour les patients âgées dont le fardeau de la maladie est mécaniquement faible. Cette proposition se traduit cependant par une perte de lisibilité de la fonction objectif dont le distribuendum est lui-même défini à partir d'un choix éthique (représenté par le choix de la fonction f)

Cette proposition est cependant $a d$-hoc et fragile. Elle n'a pas trouvé place dans les pratiques du NICE. La nature différente des distribuendum rend fondamentalement problématique la prise en compte simultanée des deux objectifs redistributifs.

L'intérêt premier du formalisme par la fonction de bien-être social est d'expliciter les présupposés éthiques du choix social du planificateur. Dans le cas présent il permet de mettre en évidence la tension entre l'objectif du fair innings et de celui de la priorisation des patients 
sévères à l'espérance de vie en bonne santé réduite. Cette tension est une réalité de I'utilisation par la société du calcul économique en santé qui n'a pas trouvé aujourd'hui d'issue claire.

\subsection{L'utilisation d'un distribuendum élargi}

Les critères de décision, tels que le critère de sévérité et de fardeau de la maladie, font l'objet de critiques de la part d'auteurs selon qui la mise en œuvre de principes égalitaristes en matière d'allocation des ressources en santé passe par un élargissement du distribuendum traditionnellement retenu dans les évaluations des stratégies de santé (Sen 2002, p.664-665). Il s'agit selon eux de ne pas se limiter à répartir équitablement de la santé, mais de répartir des conditions de vie bonnes, appréhendées de façon globale.

- L'approche par les capabilités.

Dans le prolongement des biens premiers de Rawls (1971) Sen propose de mesurer l'impact des interventions sur l'éventail des activités qu'un individu a la possibilité de mener (qu'il appelle capabilités), élémentaires (se nourrir correctement, être en bonne santé) ou complexes (participer à la vie de la communauté, avoir une haute estime de soi) (Sen 1987). Le distribuendum est alors défini de façon objective et non subjective, comme le proposent les théories utilitaristes qui évaluent l'impact des interventions sur le niveau de satisfaction personnelle (utilité). Les outils mobilisés dans le calcul économique en santé, en particulier dans le cadre des analyses coût/QALY, s'écartent déjà des théories utilitaristes puisqu'elles ne prennent en compte que partiellement les préférences individuelles et sous des conditions très restrictives (Pliskin J. et al., 1980). Sen, quant à lui, recommande de définir de façon délibérative la liste des capabilités auxquels les individus doivent avoir un égal accès. Celle-ci est toutefois susceptible de varier en fonction des spécificités des contextes locaux. Les réflexions de Sen ont particulièrement influencé les travaux récents sur la prise en compte des enjeux d'équité dans l'évaluation des interventions de santé. Coast et al., ont ainsi développé une série de questionnaires permettant d'évaluer l'impact des interventions sur les capabilités (Coast et al., 2008 ; Al-Janabi ey al. 2012) et le NICE recommande aujourd'hui leur utilisation, en particulier dans le secteur médico-social (NICE, 2016). Chaque questionnaire est adapté à une population particulière: les adultes (ICECAP-A), les personnes âgées (ICECAP-O), les aidants (CES) et les patients en fin de vie (ICECAP SCM). L'utilisation de ces questionnaires est très similaire à celle du questionnaire EQ-5D utilisé dans le calcul des QALY. Ils sont soumis à des échantillons de patients, bénéficiant ou non de l'intervention, afin qu'ils puissent décrire leur situation sur plusieurs dimensions (l'attachement, la stabilité, l'accomplissement, la joie et l'autonomie pour ICECAP-A). Pour chaque dimension, il existe plusieurs niveaux possibles allant de " pas de capabilité » à " pleine capabilité " (4 niveaux pour ICECAP-A). Des fonctions de pondération (ou fonction d'utilité) sont ensuite utilisées pour agréger les notes sur chaque dimension en une valeur unique représentant le niveau de capabilités dont bénéficient les individus ${ }^{10}$ (Flynn et al., 2015). Il est alors possible de pondérer les années de vie gagnée par le niveau de capabilité de façon à pouvoir estimer un ratio différentiel coût/année de vie en pleine capabilité (Goranitis et al. 2016). Des fonctions de pondérations ont été développées par ailleurs pour donner davantage de priorité aux personnes dont le niveau de capabilité est le plus bas, leur utilisation conduit à estimer un ratio différentiel coût/années de vie avec un niveau de capabilité suffisant (Mitchell, 2015).

\footnotetext{
10 Les fonctions de pondération sont estimées à partir des préférences des individus vis-à-vis de chaque dimension mesurée au moyen de méthode d'évaluation des choix discrets.
} 
- L'approche par le revenu équivalent santé

Une deuxième voie de recherche est offerte par Fleurbaey qui propose d'allouer les ressources de façon à répartir équitablement ce qu'il appelle les "réalisations sociales fondamentales ". II s'agit des résultats effectifs (et non des opportunités) sur les dimensions que la collectivité considère comme étant de sa responsabilité de garantir à tous de façon équitable, tels que la santé, le niveau de vie, l'éducation, l'insertion professionnelle, etc. sachant que d'autres dimensions peuvent être considérées comme relevant de la sphère privée des individus (Fleurbaey, 1995). La situation des individus sur chacune de ces dimensions peut alors être évaluée au moyen d'un indice de bien-être, le revenu équivalent. Ainsi, pour évaluer la situation effective des individus sur la dimension santé, Fleurbaey propose d'évaluer le "revenu équivalent santé "(Fleurbaey, 2011). Le revenu équivalent correspond au revenu qui donnerait à l'individu la même satisfaction s'il était combiné à une parfaite santé, que le revenu mensuel actuel de l'individu. Si un individu est en parfaite santé, son revenu équivalent santé est en principe identique à son revenu mensuel. En revanche, $s^{\prime} i l$ est en mauvais état de santé et qu'il accorde de l'importance à sa santé, sa disposition à payer pour être en parfaite santé augmente et son revenu équivalent santé devient inférieur à son revenu mensuel (Fleurbaey, 2007). Une première étude a récemment montré qu'il était empiriquement possible de réaliser une évaluation économique au moyen de cette approche dans le contexte particulier de l'évaluation des antihypertenseurs (Samson et al. 2017). Cette étude s'appuie sur les résultats d'une enquête réalisée auprès de 3331 individus en population générale, interrogés sur leur revenu mensuel, les événements de santé expérimentés durant les 12 derniers mois et leur revenu équivalent santé (mesuré à partir des consentements à payer pour avoir été en parfaite santé plutôt que dans l'état qu'ils ont décrit). Le travail de l'évaluateur consiste alors à simuler la survenue d'événements de santé dans l'échantillon, pour chaque stratégie évaluée, compte-tenu des données cliniques sur l'efficacité des traitements et l'histoire naturelle de la maladie. Il s'agit ensuite de simuler les revenus équivalent santé qu'auraient déclaré les individus en fonction de la survenue de ces événements, compte tenu de leurs préférences en termes d'arbitrage santé/revenu. Les coûts associés aux traitements et à la prise en charge des événements sont également pris en compte via l'augmentation ou la réduction des revenus des patients et des contribuables conformément aux modalités actuelles de financements des dépenses de santé. Ce travail permet ainsi d'estimer la distribution des revenus équivalent associée à chaque stratégie comparée et d'identifier celle qui permet de maximiser le bien-être total. Dans cette étude, différents degrés d'aversion aux inégalités étaient testés de façon à donner plus ou moins de valeur à l'augmentation des revenus équivalent les plus bas. Etant donné que les bénéfices sont mesurés de façon monétaire, l'approche par le revenu équivalent permet d'éviter de devoir définir une valeur seuil. Comme dans la section précédente, nous présentons de façon formelle, dans l'encadré 4, l'approche par le revenu équivalent santé afin de mettre en évidence les spécificités de l'approche comparativement au calcul économique en santé courant.

Encadré 4 : Ll'approche par les capabilités et par le revenu équivalent santé dans le calcul économique en santé

Formellement, raisonner en capabilités ou en utilités liées à la santé ne modifie par l'approche formelle décrite dans la section précédente : l'utilité liée à la santé $q_{i}$ devient capabilité mais l'écriture des fonctions de bienêtre social demeure inchangée. On reste dans un cadre où le revenu n'est pas explicitement intégré à la valorisation des situations individuelles.

Evaluer une intervention en santé en recourant à une fonction de bien-être social définie sur la santé seule n'est pas sans poser questions. Cela pose tout d'abord un problème de cohérence avec le cadre welfariste 
standard de l'analyse coût-bénéfice. En effet, dans ce cadre d'analyse confronter le gain en bien-être lié à la santé à une disposition sociétale à payer ne fait sens que si l'on impose aux fonctions d'utilité individuelle une forme de séparabilité forte entre santé et autres consommations, synthétisée dans le revenu ${ }^{11}$. Plus fondamentalement, ne pas considérer simultanément santé et revenu dans la mesure du bien-être individuel ne permet pas de saisir précisément les inégalités c'est-à-dire de considérer, au niveau individuel, les conséquences d'un état de santé dégradé, ou du financement d'un nouveau traitement, sur les revenus des individus.

Pour s'affranchir de ces limites Fleurbaey propose une mesure d'une utilité individuelle définie sur l'état de santé et le revenu: $h\left(q_{i}^{t}, y_{i}^{t}\right)$ où $y_{i}^{t}$ désigne le revenu de l'individu $i$ à la date $t$ (Fleurbaey 2007, 2011, Samson, 2017). Cette mesure est fondée sur l'index du revenu équivalent $e_{i}^{t}$ défini implicitement par l'identité suivante $: h\left(q_{i}^{t}, y_{i}^{t}\right)=h\left(\bar{q}, e_{i}^{t}\right)$ où $\bar{q}$ définit le bon état de santé. Le revenu équivalent est donc le revenu qui donne le même niveau d'utilité que l'utilité réellement observée dans la situation virtuelle où l'individu $i$ serait en bon état de santé.

L'utilité intertemporelle est alors définie comme la somme des revenus équivalents ${ }^{12} w\left(q_{i}, y_{i}\right)=\sum_{t} e_{i}^{t}$. La fonction de bien-être social associée rendant compte de l'aversion aux inégalités se définit alors ainsi: $W_{w}(q, y)=\sum_{i} f\left(w\left(q_{i}, y_{i}\right)\right)$ pour $f$ croissante concave. Cette écriture a l'avantage de considérer les inégalités de bien-être et non les seules inégalités d'utilité liée à la santé. Elle permet en outre d'intégrer l'ensemble des effets des états de santé et des interventions sur les revenus individuels (perte de productivité, financement des interventions) et plus largement de raisonner dans un cadre welfariste au sens fort du terme en évaluant l'impact d'une intervention sur le bien-être social sans avoir à valoriser socialement les gains en utilité liés à la santé. On s'éloigne alors du cadre classique du calcul économique en santé pour se rapprocher du cadre standard de l'évaluation coût-bénéfice.

\section{Conclusion}

Notre analyse avait pour objectif de rapporter les critères de priorisation mobilisés par les agences pour moduler l'utilisation des ratios coût/résultat aux principes normatifs de l'économie du bien-être, en particulier les critères de sévérité et de fardeau de la maladie. Pour cela nous avons discuté des principes de répartition induits par les deux critères et des distribuendum retenus. Il est apparu que la priorité accordée aux patients les plus sévèrement atteints ou ceux dont l'espérance de vie est la plus réduite impliquait un objectif d'égalisation des états de santé ou des durées de vie. Cet objectif contraste avec l'objectif de maximisation de la somme des gains individuels en santé sur lequel repose l'évaluation économique lorsqu'elle consiste à confronter pour un traitement donné le coût par QALY gagné au consentement à payer de la société. Le distribuendum retenu par les critères de sévérité et de fardeau reste par ailleurs restreint à des dimensions exclusivement sanitaires : l'espérance de vie et/ou la qualité de vie lié à la santé. Ces deux critères de priorité n'ont en effet pas pour objectif d'évaluer la situation des personnes sur l'ensemble des dimensions de leur vie. Des outils opérationnels ont pourtant été proposés pour prendre en compte un distribuendum élargi dans l'évaluation économique en santé, comme le questionnaire de qualité de vie ICEACP qui s'inscrit dans le cadre d'une approche par les capabilités ou le revenu équivalent santé proposé par Fleurbaey.

Mobiliser l'économie normative pour analyser les enjeux soulevés par les critères de priorisation des agences, comme nous l'avons fait dans le cadre de cet article, présente selon nous deux intérêts.

\footnotetext{
${ }^{11}$ Voir Klose (1998) par exemple pour une discussion approfondie sur ce point.

12 Nous omettons ici également l'actualisation par souci de simplification de la présentation
} 
En premier lieu, cette approche est utile pour organiser les discussions. Elle permet de distinguer les notions les unes par rapport aux autres et de mettre en lumière les principes de justice sous-jacents. Elle permet également de discuter la compatibilité entre certaines notions, et dans d'autres cas, leurs contradictions. Nous pensons donc que cet exercice est une étape utile pour faire avancer les discussions au sein des institutions sur les critères d'évaluation des interventions de santé et les procédures de décision.

En second lieu, la formalisation des fonctions-objectifs adaptés aux impératifs éthiques qui orientent aujourd'hui les décisions des agences met en lumière les perspectives possibles pour amender le calcul économique. Le résultat de ces évaluations économiques égalitaristes pourrait être porté à la connaissance des décideurs, en complément des évaluations économiques classiques. Aujourd'hui les agences se limitent à pondérer qualitativement les résultats des évaluations économiques en termes de ratio différentiel coût/résultat, dans le cadre des délibérations, par ces critères de priorisation. Documenter quantitativement les conséquences des interventions de santé du point de vue de l'objectif d'égalisation des états de santé, des durées de vie ou des conditions de vie globales des individus permet d'accroitre l'intelligibilité des enjeux par l'ensemble des acteurs et donc de favoriser la transparence et cohérence des décisions. L'intérêt qu'il y a à nourrir les délibérations d'évaluations quantitatives des conséquences des décisions publiques est justifié, y compris dans le cadre de modèle de justice procédurale, comme souligné par Norman Daniels dans son ouvrage Just health (2007).

\section{Bibliographie}

Al-Janabi H, Flynn T, Coast J., (2012), Development of a self-report measure of capability wellbeing for adults: the ICECAP-A. Quality of Life Research 21: 167-176.

Asaria M, Griffin S, Cookson R, et al., (2015), Distributional cost-effectiveness analysis of health care programmes - a methodological case study of the UK bowel cancer screening programme. Health Economics 24(6):742-754.

Binder-Foucard F, Belot A, Delafosse P, et al., (2013), Estimation nationale de l'incidence et de la mortalité par cancer en France entre 1980 et 2012.

Brazier J., Ratcliffe J., Salomon J., Tsuchiya A. 2007. Measuring and Valuing Health Benefits for Economic Evaluation, Oxford University Press, $2^{\text {nd }}$ edition

Brazier J., Rowena D., Mukuriaa C., et al., (2013), Eliciting societal preferences for burden of illness, therapeutic improvement and end of life for value based pricing: a report of the main survey, Research report

Cleemput I, Neyt N, de Laet C et al., (2008), Valeurs seuils pour le rapport coût-efficacité en soins de santé. KCE reports $100 \mathrm{~B}$

Cleemput I., Stephan D., Kohn L. et al., (2014), Comment prendre en compte les préférences des citoyens dans la décision de rembourser un nouveau traitement, Reflets et perspectives de la vie économique, 4 (Tome LIII), p. 35-53.

Clément, V. (2009). Economie du bien-être, choix social et l'influence de la Théorie de la justice. Raisons politiques, 33,(1), 57-79.

Coast J, Smith R, Lorgelly P., (2008), Should the capability approach be applied in health economics? Health Economics 17:667-670.

College voor zorgverzekerinen (CVZ), (2010), A background study on the "cost-effectiveness" package principle for the benefit of the appraisal phase in package management. 
Collins M., Latimer N., (2013), NICE's end of life criteria: who gains, who loses? BMJ, 346

Cookson R, Mirelman AJ, Griffin S, et al., (2017), Using Cost-Effectiveness Analysis to Address Health Equity Concerns. Value in Health ; 20(2):206-212.

Cour des comptes, (2011), Rapport sur l'application des lois de financement de la sécurité sociale, Chapitre IV La maîtrise des dépenses de médicaments

Culyer A, Wagstaff A., (1993), Equity and equality in health and health care. J Health Econ.

Dec;12(4):431-57.

Daniel N., (2007), Just health : Meeting Health Needs Fairly, Cambridge university Press

DREES, (2016), La protection sociale en France et en Europe en 2014

Dormont B., (2009), Les Dépenses de Santé Une augmentation salutaire ? Edition rue d'Ulm, centre pour la recherche économique et ses applications

Fleurbaey M., (1995), EQual opportunity of equal social outcome, Economics and

philosophy. 11. 25-55

Fleurbaey M., (1996), Théories économiques de la justice. Paris: Economica

Fleurbaey M., (2003), Ni perfectionniste ni welfariste: l'indice des biens premiers est possible", Revue de Philosophie Economique no 7: 111-135.

Fleurbaey M., (2011), Willingness-to-pay and the equivalence approach, Revue d'économie politique, 1 (Vol. 121), p. 35-58

Flynn TN, Huynh E, Peters TJ, et al. (2015) Scoring the Icecap-A Capability Instrument. Estimation of a UK General Population Tariff. Health Economics ; 24(3):258-269.

Forget E., (2004), Contested Histories of an Applied Field: The Case of Health Economics, History of Political Economy 36(4): 617-38

Golan 01, Hansen P, Kaplan G et al., (2011), Health technology prioritization: which criteria for prioritizing new technologies and what are their relative weights? Health Policy. Oct;102(23):126-35

Goranitis I., Coast J., Day E., et al., (2016), Maximizing Health or Sufficient Capability in Economic Evaluation? A Methodological Experiment of Treatment for Drug Addiction, Medical Decision Making.

Harris, J., (1985), The Value Of Life. Routledge.

Haute Autorité de Santé, (2014), Valeurs de références pour l'évaluation économique en santé: Revue de la littérature

Hurka T., (2004), Article " Perfectionisme ", in Canto-Sperber M., Dictionnaire d'éthique et de philosophie morale, PUF

Institut national des cancers (INCA), (2015), Situation de la chimiothérapie des cancers : année 2014

Kusel J., (2015), Why Has Value Based Assessment Been Abandoned by NICE in the UK?, september/october

Midy F., Raimond V., Thébaut C., et al., (2015), Avis d'efficience relatifs aux produits de santé à la Haute Autorité de santé : bilan et perspectives, Santé Publique, Vol. 27

Miners A., Cairns J. et Wailoo A. (2013). Department of Health Proposals for Including Burden of Illness into Value Based Pricing: A Description and Critique, Nice Decision Support Unit Mitchell P., Roberts T., Barton P., et al., (2015), Assessing Sufficient Capability: A New Approach to Economic Evaluation. Soc Sci Med 139, 71-79. Jun 30.

National Institute for Health and Clinical Excellence (NICE), (2008), Social value judgements. Principles for the development of NICE guidance. 2nd edition. London

National Institute for Health and Clinical Excellence, (2009), Appraising life-extending, end of life treatments. 
National Institute for Health and Clinical Excellence, (2014), Value Based Assessment of Health Technologies (Consultation Paper).

National Institute for Health and Clinical Excellence, (2016), The social care guidance manual Nord E, Pinto J, Richardson J et al. (1999) Incorporating societal concerns for fairness in numerical valuations of health programmes. Health Econ ;8(1):25-39.

Pharmaceutical Benefits Advisory Committee (PBAC), (2013), Guidelines for preparing submissions to the Pharmaceutical Benefits Advisory Committee. Version 4.4

Pajares y Sanchez C. et Saout C., (2017), Prix et accès aux traitements médicamenteux innovants, Avis du Conseil économique, social et environnemental

Pliskin, J., Shepard, D. and Weinstein, M., (1980). Utility Functions For Life Years And Health Status, Operation Research, 28, 206-244.

Raftery J., (2009), Should NICE's threshold range for cost per QALY be raised? No. BMJ;338:b185

Rawls J., (1971), Théorie de la justice, Le Seuil, Point, 1997

Sabik LM, Lie RK. ,(2008), Priority setting in health care: lessons from the experiences of eight countries. Int J Equity Health;7:4.

Samson A-L., Schokkaert E., Thébaut C., et al., (2017), Fairness in cost-benefit analysis: an application to health technology assessment, Health Economics. 2017;1-13.

Sen A., (1987), On ethics and economics. Oxford: Basil Blackwell.

Towse A., Barnsley P., (2013), Clarifying meanings of absolute and proportional shortfall with examples, Office of Health Economics

Tsuchiya A., (2001), The Value of Health at Different Ages, Discussion paper 184, University of York

Van Parijs P., (1991), Qu'est-ce qu'une société juste ? Introduction à la pratique de la philosophie politique. Paris: Seuil;

Weinstein MC. A QALY is a QALY--or is it? J Health Econ. 1988 Sep;7(3):289-90

Raftery, J., (2006) Review of NICE's recommendations, 1999-2005, BMJ. May 27; 332(7552): 1266-1268.

Van de Wetering E, Stolk E, van Exel $\mathrm{N}$ et al., (2013), Balancing equity and efficiency in the Dutch basic benefits package using the principle of proportional shortfall. Eur J Health Econ;14(1):107-15.

WHO, (2013), methods and data sources for global burden of disease estimates 2000-2011. Williams A., (1997) Intergenerational equity: an exploration of the 'fair innings' argument, Health Economics, vol. 6: 117-132

Wailoo A., Tsuchiya A., McCabe C., (2009), Weighting must wait. Pharmacoeconomics. 27:983-989. 